\title{
The Approximation Szász-Chlodowsky Type Operators Involving Gould-Hopper Type Polynomials
}

\author{
Behar Baxhaku and Artan Berisha \\ Department of Mathematics, University of Prishtina, Mother Teresa, 10000 Prishtina, Kosovo \\ Correspondence should be addressed to Artan Berisha; artan.berisha@uni-pr.edu
}

Received 23 March 2017; Accepted 18 June 2017; Published 26 July 2017

Academic Editor: Jaume Giné

Copyright (c) 2017 Behar Baxhaku and Artan Berisha. This is an open access article distributed under the Creative Commons Attribution License, which permits unrestricted use, distribution, and reproduction in any medium, provided the original work is properly cited.

\begin{abstract}
We introduce the Szász and Chlodowsky operators based on Gould-Hopper polynomials and study the statistical convergence of these operators in a weighted space of functions on a positive semiaxis. Further, a Voronovskaja type result is obtained for the operators containing Gould-Hopper polynomials. Finally, some graphical examples for the convergence of this type of operator are given.
\end{abstract}

\section{Introduction}

In [1], Jakimovski and Leviatan introduced and investigated some approximation properties of the Favard-Szász type operator, by using Appell polynomials $p_{k}(x) \geq 0$ which satisfy the identity

$$
g(t) e^{t x}=\sum_{k=0}^{\infty} p_{k}(x) t^{k}
$$

where $g(z)=\sum_{k=0}^{\infty} a_{k} z^{k}$ is an analytic function in the disc $|z|<R,(R>1)$ and $g(1) \neq 0$,

$$
J(f ; x)=\frac{e^{n x}}{g(1)} \sum_{k=0}^{\infty} p_{k}(n x) f\left(\frac{k}{n} b_{n}\right), \quad x \in[0, \infty) .
$$

Varma et al. constituted a link between orthogonal polynomials and the positive linear operators. In [1], they proposed Szász operators involving the Brenke polynomials defined by

$$
T_{n}(f ; x)=\frac{1}{A(1) B(n x)} \sum_{k=0}^{\infty} p_{k}(n x) f\left(\frac{k}{n}\right),
$$

where $x \geq 0$ and $n \in \mathbb{N}$.

Recently, Büyükyazıcı et al. in [2] introduced the Chlodowsky variant of operators (3). Inspired by this work, we give the Szász-Chlodowsky type operators including Gould-Hopper polynomials. The generating functions for these Gould-Hopper polynomials are given by

$$
e^{h t^{d+1}} e^{x t}=\sum_{k=0}^{\infty} g_{k}^{d+1}(x, h) \frac{t^{k}}{k !}
$$

and the explicit representations

$$
g_{k}^{d+1}(x, h)=\sum_{k=0}^{[k / d+1]} \frac{k !}{s !(k-(d+1) s) !} h^{s} x^{k-(d+1) s},
$$

where [-] denotes the integer part. Now, let us define SzászChlodowsky type generalization of the Szász operators with the help of generating function (4), as follows:

$$
\begin{array}{r}
\mathscr{G}_{n, h}^{(d)}(f ; x)=e^{-n x / b_{n}-h} \sum_{k=0}^{\infty} \frac{g_{k}^{d+1}\left(n x / b_{n}, h\right)}{k !} f\left(\frac{k}{n} b_{n}\right), \\
x \in[0, \infty),
\end{array}
$$

where $h \geq 0$ and $b_{n}$ is a positive increasing sequence with the properties

$$
\begin{aligned}
& \lim _{n \rightarrow \infty} b_{n}=\infty, \\
& \lim _{n \rightarrow \infty} \frac{b_{n}}{n}=0 .
\end{aligned}
$$


The generalization of Szász type operators has been studied in $[3-10]$.

The aim of the this paper is to study some direct results in terms of the modulus of continuity of the second order, convergence of derivative operators to derivative functions, the weighted space, and the degree of approximation of $f$ by $\mathscr{G}_{n, h}^{(d)}$. We also study the statistical convergence. The rate of convergence of the operators $\mathscr{G}_{n, h}^{(d)}$ to a certain function is also illustrated through graphics using Matlab.

\section{Notations and Auxiliary Results}

Let us denote $C_{E}[0, \infty):=\left\{f \in C[0, \infty):|f(t)| \leq M e^{N t}, t \in\right.$ $[0, \infty)$, for some $M, N>0\}$. The following notations and lemmas are needed to prove the main results.

In what follows, let $e_{i}(t)=t^{i}, i \in N^{0}$, be the test functions.

Lemma 1. From (4), one has

(i) $\sum_{k=0}^{\infty}\left(g_{k}^{d+1}\left(n x / b_{n}, h\right) / k !\right)=e^{n x / b_{n}+h}=\kappa_{1}(x)$;

(ii) $\sum_{k=0}^{\infty}\left(k g_{k}^{d+1}\left(n x / b_{n}, h\right) / k\right.$ !) $=e^{n x / b_{n}+h}\left(n x / b_{n}+h(d+\right.$ $1))=\kappa_{2}(x)$

(iii) $\sum_{k=0}^{\infty}\left(k^{2} g_{k}^{d+1}\left(n x / b_{n}, h\right) / k !\right)=e^{n x / b_{n}+h}\left(n^{2} x^{2} / b_{n}^{2}+(n x /\right.$ $\left.\left.b_{n}\right)(2 h(d+1)+1)+h(d+1)^{2}(h+1)\right)=\kappa_{3}(x)$;

(iv) $\sum_{k=0}^{\infty}\left(k^{3} g_{k}^{d+1}\left(n x / b_{n}, h\right) / k !\right)=e^{n x / b_{n}+h}\left(n^{3} x^{3} / b_{n}^{3}+\right.$ $\left(3 n^{2} x^{2} / b_{n}^{2}\right)(h(d+1)+1)+\left(3 n x / b_{n}\right)(h(d+1)(h(d+$ $\left.1)+d+2)+1 / 3)+h(d+1)^{3}\left(h^{2}+3 h+1\right)\right)=\kappa_{4}(x)$;

(v) $\sum_{k=0}^{\infty}\left(k^{4} g_{k}^{d+1}\left(n x / b_{n}, h\right) / k !\right)=e^{n x / b_{n}+h}\left(n^{4} x^{4} / b_{n}^{4}+\right.$ $\left(2 n^{3} x^{3} / b_{n}^{3}\right)(2 h(d+1)+3)+\left(6 n^{2} x^{2} / b_{n}^{2}\right)\left(h^{2}(d+1)^{2}+\right.$ $h(d+1)(d+3)+7 / 6)+\left(2 n x / b_{n}\right)\left(3 h^{2}(d+1)^{2}(2 d+3)+\right.$ $\left.h(d+1)\left(2 d^{2}+7 d+7\right)+2 h^{3}(d+1)^{3}+1 / 2\right)+h(d+$ $\left.1)^{4}\left(h^{3}+6 h^{2}+7 h+1\right)\right)=\kappa_{5}(x)$.

Lemma 2. For the operators $\mathscr{G}_{n, h}^{(d)}$, one has

(i) $\mathscr{G}_{n, h}^{(d)}\left(e_{0} ; x\right)=1$;

(ii) $\mathscr{G}_{n, h}^{(d)}\left(e_{1} ; x\right)=x+\left(b_{n} / n\right) h(d+1)$;

(iii) $\mathscr{G}_{n, h}^{(d)}\left(e_{2} ; x\right)=x^{2}+\left(b_{n} x / n\right)(2 h(d+1)+1)+\left(b_{n}^{2} / n^{2}\right) h(h+$ 1) $(d+1)^{2}$;

(iv) $\mathscr{G}_{n, h}^{(d)}\left(e_{3} ; x\right)=x^{3}+\left(3 b_{n} x^{2} / n\right)(h(d+1)+1)+\left(3 b_{n}^{2} x /\right.$ $\left.n^{2}\right)(h(d+1)(h(d+1)+d+2)+1 / 3)+\left(3 b_{n}^{3} / n^{3}\right) h(d+$ $1)^{2}\left((d+1)\left(h^{2}+1\right)+h(2 d+1)\right)$;

(v) $\mathscr{G}_{n, h}^{(d)}\left(e_{4} ; x\right)=x^{4}+\left(2 b_{n} x^{3} / n\right)(2 h(d+1)+3)+\left(6 b_{n}^{2} x^{2} /\right.$ $\left.n^{2}\right)\left(h^{2}(d+1)^{2}+h(d+1)(d+3)+7 / 6\right)+\left(2 b_{n}^{3} x /\right.$ $\left.n^{3}\right)\left(3 h^{2}(d+1)^{2}(2 d+3)+h(d+1)\left(2 d^{2}+7 d+7\right)+2 h^{3}(d+\right.$ $\left.1)^{3}+1 / 2\right)+\left(b_{n}^{4} / n^{4}\right)\left(h(d+1)^{4}\left(h^{3}+6 h^{2}+7 h+1\right)\right)$.

Proof. From Lemma 2 and by definition of $\mathscr{G}_{n, h}^{(d)}$, we have

$$
\begin{aligned}
& \mathscr{G}_{n, h}^{(d)}\left(e_{0} ; x\right)=e^{-n x / b_{n}-h_{1}} \kappa_{1}(x)=1, \\
& \mathscr{G}_{n, h}^{(d)}\left(e_{1} ; x\right)=\frac{b_{n}}{n} e^{-n x / b_{n}-h} \kappa_{2}(x)=x+\frac{b_{n}}{n} h(d+1) .
\end{aligned}
$$

Now, we consider the case $\mathscr{G}_{n, h}^{(d)}\left(e_{2} ; x\right)$ as follows:

$$
\begin{aligned}
\mathscr{G}_{n, h}^{(d)}\left(e_{2} ; x\right)= & \frac{b_{n}^{3}}{n^{3}} e^{-n x / b_{n}-h} \kappa_{4}(x) \\
= & x^{2}+\frac{b_{n} x}{n}(2 h(d+1)+1) \\
& +\frac{b_{n}^{2}}{n^{2}} h(h+1)(d+1)^{2} .
\end{aligned}
$$

For $e_{3}$, we have

$$
\begin{aligned}
\mathscr{G}_{n, h}^{(d)}\left(e_{3} ; x\right) & =\frac{b_{n}^{2}}{n^{2}} e^{-n x / b_{n}-h} \kappa_{3}(x) \\
= & x^{3}+\frac{3 b_{n} x^{2}}{n}(h(d+1)+1) \\
& +\frac{3 b_{n}^{2} x}{n^{2}}\left(h(d+1)(h(d+1)+d+2)+\frac{1}{3}\right) \\
& +\frac{3 b_{n}^{3}}{n^{3}} h(d+1)^{2}\left((d+1)\left(h^{2}+1\right)+h(2 d+1)\right),
\end{aligned}
$$

and, finally,

$$
\begin{aligned}
& \mathscr{G}_{n, h}^{(d)}\left(e_{4} ; x\right)=\frac{b_{n}^{4}}{n^{4}} e^{-n x / b_{n}-h} \kappa_{5}(x)=x^{4} \\
& +\frac{2 b_{n} x^{3}}{n}(2 h(d+1)+3)+\frac{6 b_{n}^{2} x^{2}}{n^{2}}\left(h^{2}(d+1)^{2}\right. \\
& \left.+h(d+1)(d+3)+\frac{7}{6}\right) \\
& +\frac{2 b_{n}^{3} x}{n^{3}}\left(3 h^{2}(d+1)^{2}(2 d+3)\right. \\
& \left.+h(d+1)\left(2 d^{2}+7 d+7\right)+2 h^{3}(d+1)^{3}+\frac{1}{2}\right) \\
& +\frac{b_{n}^{4}}{n^{4}}\left(h(d+1)^{4}\left(h^{3}+6 h^{2}+7 h+1\right)\right) .
\end{aligned}
$$

Lemma 3. Let $\eta_{n, s}^{d}(x)=\mathscr{G}_{n, h}^{(d)}\left(\left(e_{1}-x\right)^{s} ; x\right)$, and then, for every $x \in[0, \infty)$, one has

(i) $\eta_{n, 1}^{(d)}(x)=\left(b_{n} / n\right) h(d+1)$;

(ii) $\eta_{n, 2}^{(d)}(x)=b_{n} x / n+\left(b_{n}^{2} / n^{2}\right) h(h+1)(d+1)^{2}$;

(iii) $\eta_{n, 4}^{(d)}(x)=3 b_{n}^{2} x^{2} / n^{2}+\left(2 b_{n}^{3} x / n^{3}\right)\left((d+1)^{3} h\left(-2 h^{2}+3 h-\right.\right.$ $\left.3)+h(d+1)^{2}\left(-3 h+7-12 h d+2 d^{2}\right)+1 / 2\right)+\left(b_{n}^{4} / n^{4}\right) h(d+$ $1)^{4}\left(h^{3}+6 h^{2}+7 h+1\right)$.

Theorem 4. Let $f \in C_{E}[0, \infty)$. Then, $\lim _{n \rightarrow \infty} \mathscr{G}_{n, h}^{(d)}(f ; x)=$ $f(x)$, uniformly on each compact subset of $[0, \infty)$.

Proof. By Lemma 2, $\lim _{n \rightarrow \infty} \mathscr{G}_{n, h}^{(d)}\left(e_{i} ; x\right), i=0,1,2$, uniformly on every compact subset of $[0, \infty)$. So, by Bohman-Korovkin theorem, the result follows. 


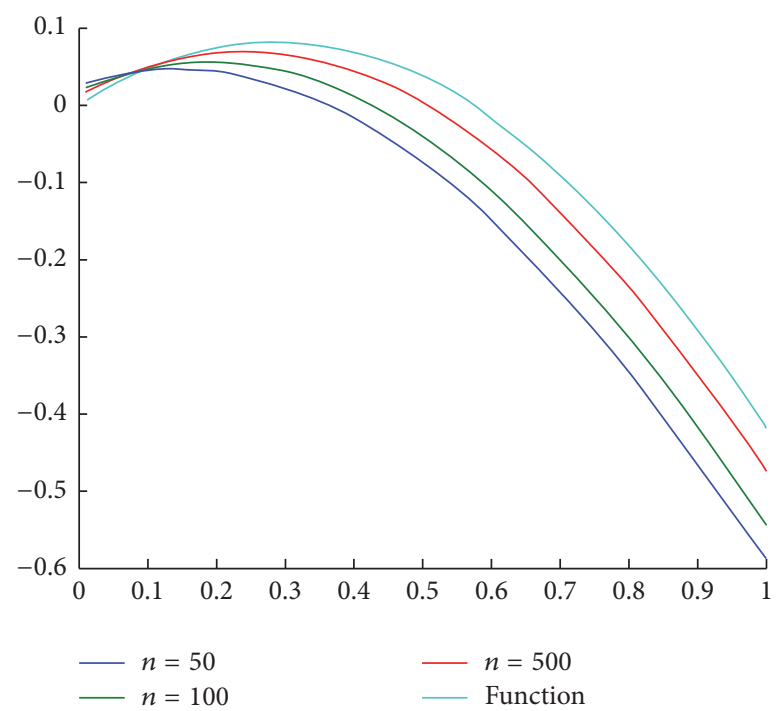

(a)

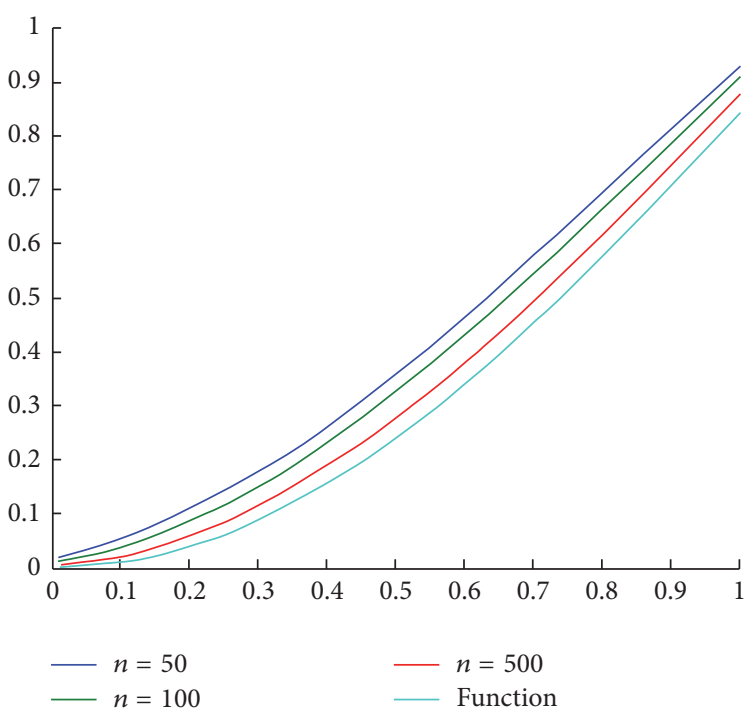

(b)

Figure 1

Example 5. For $n=50,100$, and 500, $d=0.5$, and $b_{n}=$ $\sqrt{n}$, the convergence of $\mathscr{G}_{n, h}^{(d)}(f ; x)$ to $f(x)=x \cos (x+1)$ is illustrated in Figure 1(a).

Example 6. For $n=50,100$, and 500, $d=0.5$, and $b_{n}=$ $\sqrt{n}$, the convergence of $\mathscr{G}_{n, h}^{(d)}(f ; x)$ to $f(x)=x^{2} / \sqrt{x^{2}+1}$ is illustrated in Figure 1(b).

Theorem 7. Let $f \in C_{E}[0, \infty)$, and then for any $x \in[0, c]$ one has

$$
\begin{aligned}
& \left|\mathscr{G}_{n, h}^{(d)}(f ; x)-f(x)\right| \\
& \quad \leq\left\{1+\sqrt{c+\frac{b_{n}}{n} h(h+1)(d+1)^{2}}\right\} \omega\left(f ; \sqrt{\frac{b_{n}}{n}}\right) .
\end{aligned}
$$

Proof. By using Lemma 3 case (ii) and the well-known properties of the modulus of continuity, we have

$$
\begin{aligned}
& \left|\mathscr{G}_{n, h}^{(d)}(f ; x)-f(x)\right| \leq \mathscr{G}_{n, h}^{(d)}\left(\left|f\left(\frac{k}{n} b_{n}\right)-f(x)\right| ; x\right) \\
& \quad \leq\left\{1+\frac{1}{\delta} \mathscr{G}_{n, h}^{(d)}\left(\left|\frac{k}{n} b_{n}-x\right| ; x\right)\right\} \omega(f ; \delta) .
\end{aligned}
$$

Recalling the Cauchy-Schwarz inequality, we obtain the formula below:

$$
\begin{aligned}
& \left|\mathscr{G}_{n, h}^{(d)}(f ; x)-f(x)\right| \\
& \quad \leq\left\{1+\frac{1}{\delta}\left(\mathscr{G}_{n, h}^{(d)}\left(\left(\frac{k}{n} b_{n}-x\right)^{2} ; x\right)\right)^{1 / 2}\right\} \omega(f ; \delta) \\
& \quad=\left\{1+\frac{1}{\delta} \sqrt{\mathscr{G}_{n, h}^{(d)}\left((t-x)^{2} ; x\right)}\right\} .
\end{aligned}
$$

By means of Lemma 3 case (ii), for $0 \leq x \leq c$, one gets

$$
\mathscr{G}_{n, h}^{(d)}\left(\left(e_{1}-x\right)^{2} ; x\right) \leq \frac{b_{n} c}{n}+\frac{b_{n}^{2}}{n^{2}} h(h+1)(d+1)^{2} .
$$

Using (22) and taking $\delta=\sqrt{b_{n} / n}$ in (19), we obtain the desired result.

The theorem below shows that the derivative $\left(d^{r} / d x^{r}\right) \mathscr{G}_{n, h}^{(d)}(f ; x)$ is also an approximation process for $d^{r} f / d x^{r}$.

Theorem 8. Let $f \in C_{E}[0, \infty)$. If $f^{(r)}$ exists at a point $x \in(0$, $\infty)$, then one has

$$
\begin{aligned}
& \left|\frac{d^{r}}{d x^{r}} \mathscr{G}_{n, h}^{(d)}(f ; x)-\frac{d^{r}}{d x^{r}} f(x)\right| \\
& \quad \leq r !\left\{1+\sqrt{c+\frac{b_{n}}{n} h(h+1)(d+1)^{2}}\right\} \omega\left(f ; \sqrt{\frac{b_{n}}{n}}\right) \\
& \quad+\omega\left(\frac{d^{r}}{d x^{r}} f ; r \frac{b_{n}}{n}\right),
\end{aligned}
$$

where $\omega\left(d^{r} f / d x^{r}, \cdot\right)$ is the modulus of continuity of $d^{r} f / d x^{r}$.

Proof. By simple calculations, the following formula is obtained:

$$
\frac{d^{r}}{d x^{r}} \mathscr{G}_{n, h}^{(d)}(f ; x)=\left(\frac{n}{b_{n}}\right)^{r} \mathscr{G}_{n, h}^{(d)}\left(\Delta_{b_{n} / n}^{r} f ; x\right),
$$

where $\Delta_{b_{n} / n}^{r} f\left((k / n) b_{n}\right)$ is the difference of order $r$ of $f$ corresponding to the increment $b_{n} / n$. Using the relation between 
finite difference and divided difference, the derivative of order $r$ of the operators is represented as follows:

$$
\begin{aligned}
& \frac{d^{r}}{d x^{r}} \mathscr{G}_{n, h}^{(d)}(f ; x)=r ! \mathscr{G}_{n, h}^{(d)}\left(\frac{\Delta_{b_{n} / n}^{r} f}{r !\left(b_{n} / n\right)^{r}} ; x\right) \\
& =r ! e^{-n x / b_{n}-h} \sum_{k=0}^{\infty} \frac{g_{k}^{d+1}\left(n x / b_{n}, h\right)}{k !}\left[\frac{k}{n} b_{n}, \frac{k+1}{n}\right. \\
& \left.\cdot b_{n}, \ldots, \frac{k+r}{n} b_{n} ; f\right]=r ! \mathscr{G}_{n, h}^{(d)}(\mu ; x),
\end{aligned}
$$

where $\mu(x)=\left[x, x+b_{n} / n, \ldots, x+r\left(b_{n} / n\right) ; f\right]$. Then, using Theorem 4, we have

$$
\begin{aligned}
& \left|\frac{d^{r}}{d x^{r}} \mathscr{G}_{n, h}^{(d)}(f ; x)-\frac{d^{r}}{d x^{r}} f(x)\right| \\
& \leq r !\left|\mathscr{G}_{n, h}^{(d)}(\mu ; x)-\mu(x)\right|+\left|r ! \mu(x)-\frac{d^{r}}{d x^{r}} f(x)\right| \\
& \leq r !\left\{1+\sqrt{c+\frac{b_{n}}{n} h(h+1)(d+1)^{2}}\right\} \omega\left(f ; \sqrt{\frac{b_{n}}{n}}\right) \\
& \quad+\left|r ! \mu(x)-\frac{d^{r}}{d x^{r}} f(x)\right| .
\end{aligned}
$$

By using the mean value theorem and some known classical properties of the modulus of continuity [11], one has

$$
\begin{aligned}
\mid \mu & (x+\delta)-\mu(x) \mid \\
& =\mid\left[x+\delta, x+\delta+\frac{b_{n}}{n}, \ldots, x+\delta+r \frac{b_{n}}{n} ; f\right] \\
& -\left[x, x+\frac{b_{n}}{n}, \ldots, x+r \frac{b_{n}}{n} ; f\right] \mid \\
& +\frac{1}{r !} \mid \frac{d^{r}}{d x^{r}} f\left(x+\delta+r \frac{b_{n}}{n} \phi_{1}\right) \\
& -\frac{d^{r}}{d x^{r}} f\left(x+r \frac{b_{n}}{n} \phi_{2}\right) \mid+\frac{1}{r !} \omega\left(\frac{d^{r}}{d x^{r}} f ; \delta\right. \\
& \left.+r \frac{b_{n}}{n}\left|\phi_{1}-\phi_{2}\right|\right) \leq \frac{1}{r !} \omega\left(\frac{d^{r}}{d x^{r}} f ; \delta+r \frac{b_{n}}{n}\right),
\end{aligned}
$$

where $\phi_{1}, \phi_{2} \in(0,1)$. Hence, we obtain

$$
\omega(\mu, \delta) \leq \frac{1}{r !} \omega\left(\frac{d^{r}}{d x^{r}} f ; \delta+r \frac{b_{n}}{n}\right)
$$

On the other hand,

$$
\begin{aligned}
& \left|r ! \mu(x)-\frac{d^{r}}{d x^{r}} f(x)\right| \\
& \quad \leq\left|r !\left[x, x+\frac{b_{n}}{n}, \ldots, x+r \frac{b_{n}}{n} ; f\right]-\frac{d^{r}}{d x^{r}} f(x)\right|
\end{aligned}
$$

$$
\begin{aligned}
& \leq\left|\frac{d^{r}}{d x^{r}} f\left(x+r \frac{b_{n}}{n} \phi_{3}\right)-\frac{d^{r}}{d x^{r}} f(x)\right| \\
& \leq \omega\left(\frac{d^{r}}{d x^{r}} f ; \phi_{3} r \frac{b_{n}}{n}\right) \leq \omega\left(\frac{d^{r}}{d x^{r}} f ; r \frac{b_{n}}{n}\right),
\end{aligned}
$$

where $\phi_{3} \in(0,1)$. Using the estimates in (15), we have the desired result.

\section{Weighted Approximation}

Let $D_{\psi}[0, \infty)$ be the space of all functions $f$ defined on $[0, \infty)$ satisfying the condition $|f(x)| \leq M_{f} \psi(x)$, where $M_{f}$ is a positive constant depending only on $f$ and $\psi(x)=1+x^{2}$ is a weight function. By $C_{\psi}[0, \infty)$, we denote the subspace of all continuous functions $f \in D_{\psi}[0, \infty)$ with the norm $\|f\|_{\psi}=\sup _{x \in[0, \infty)}\left(|f(x)| /\left(1+x^{2}\right)\right)$ and $C_{\psi}^{*}[0, \infty)=\{f \in$ $\left.C_{\psi}[0, \infty): \lim _{n \rightarrow \infty}\left(f(x) /\left(1+x^{2}\right)\right)<\infty\right\}$.

Theorem 9. Let $f \in C_{\psi}[0, \infty)$, and let $\psi=1+x^{2}$ be a weighted function; then, the inequality

$$
\left\|\mathscr{G}_{n, h}^{(d)}(\psi ; x)\right\|_{\psi} \leq 1+M_{f}
$$

is satisfied.

Proof. Using Lemma 2, one has

$$
\begin{aligned}
& \left\|\mathscr{G}_{n, h}^{(d)}(f ; x)\right\|_{\psi}=\sup _{x \geq 0} \frac{1}{1+x^{2}}\left\{1+x^{2}\right. \\
& \left.+\frac{b_{n} x}{n}(2 h(d+1)+1)+\frac{b_{n}^{2}}{n^{2}} h(h+1)(d+1)^{2}\right\} \\
& \quad \leq\left\{1+\frac{b_{n}}{n}(2 h(d+1)+1)\right. \\
& \left.+\frac{b_{n}^{2}}{n^{2}} h(h+1)(d+1)^{2}\right\} .
\end{aligned}
$$

Since $\lim _{n \rightarrow \infty}\left(b_{n} / n\right)=0$, there exists constant $M_{f}$ such that

$$
\left\|\mathscr{G}_{n, h}^{(d)}(\psi ; x)\right\|_{\psi} \leq 1+M_{f}
$$

This proof is complete.

From [12], for $f \in C_{\psi}^{*}[0, \infty)$, the weighted modulus of continuity of $f$ is defined by

$$
\begin{aligned}
& \Omega_{\psi}(f ; \delta) \\
& =\sup _{0 \leq|\psi(x)-\psi(t)|<\delta, x, t \in[0, \infty)} \frac{|f(x)-f(t)|}{[|\psi(x)-\psi(t)|+1] \psi(x)},
\end{aligned}
$$

where $\psi$ is a continuously differentiable function on $[0, \infty)$, $\psi(0)=0$, and $\inf _{x \geq 0} \psi^{\prime}(x) \geq 1$. Now, we define following 
sequence of positive linear operators $\mathscr{P}_{n, h}^{(d)}$ defined with the help of $\mathscr{G}_{n, h}^{(d)}$ defined in (6):

$$
\begin{aligned}
\mathscr{P}_{n, h}^{(d)}(f ; x) & =e^{-n x / b_{n}-h} \psi^{2}(x) \\
\cdot \sum_{k=0}^{\infty} g_{k}^{d+1}\left(\frac{n x}{b_{n}}, h\right) \frac{1}{\psi^{2}\left((k / n) b_{n}\right) k !} f\left(\frac{k}{n} b_{n}\right), & \\
x & \in[0, \infty) .
\end{aligned}
$$

Theorem 10 (see [12]). Let $\left(L_{n}\right)$ be the sequences of linear positive operators and $\eta(x) \leq \psi_{k}(x), k=1,2$, 3. If

$$
\begin{gathered}
\left\|L_{n} 1-1\right\|_{\psi_{1}}=\alpha_{n}, \\
\left\|L_{n} \rho-\rho\right\|_{\psi_{2}}=\beta_{n}, \\
\left\|L_{n} \rho^{2}-\rho^{2}\right\|_{\psi_{3}}=\gamma_{n},
\end{gathered}
$$

where $\psi(x)=\max \left\{\psi_{1}, \psi_{2}, \psi_{3}\right\}$ and $\alpha_{n}, \beta_{n}$, and $\gamma_{n}$ tend to zero as $n \rightarrow \infty$, then, for all functions $f \in C_{\psi}^{*}[0, \infty)$, the inequality

$$
\begin{aligned}
\left\|L_{n}(f ; x)-f\right\|_{\psi \rho^{2}} \leq & \Omega_{\rho}\left(f ; \sqrt{\alpha_{n}+\beta_{n}+\gamma_{n}}\right) \\
& +\|f\|_{\rho} \alpha_{n}
\end{aligned}
$$

holds for $n$ large enough.

Theorem 11. Let $\mathscr{P}_{n, h}^{(d)}$ be the sequences of linear positive operators defined by (27) and $\eta(x)=1+x^{2}$. If $f \in C_{\psi}^{*}[0, \infty)$, then the inequality

$$
\begin{aligned}
\left\|\mathscr{P}_{n, h}^{(d)}(f ; x)-f\right\|_{\psi^{4} \eta} \leq & 16 \Omega_{\psi}\left(f ; \sqrt{\alpha_{n}+2 \beta_{n}}\right) \\
& +\|f\|_{\psi} \alpha_{n}
\end{aligned}
$$

is satisfied, where $\alpha_{n}=(2 h(d+1)+1)\left(b_{n} / n\right)+\left(b_{n} / n\right)^{2} h(h+$ 1) $(d+1)^{2}$ and $\beta_{n}=\left(b_{n} / n\right) h(d+1)$.

Proof. By simple calculation, we have

$$
\begin{gathered}
\mathscr{P}_{n, h}^{(d)}(1 ; x)-1=\psi^{2}(x)\left[e^{-n x / b_{n}-h} \sum_{k=0}^{\infty} g_{k}^{d+1}\left(\frac{n x}{b_{n}}, h\right)\right. \\
\left.\cdot \frac{1}{\psi^{2}\left((k / n) b_{n}\right) k !} f\left(\frac{k}{n} b_{n}\right)-\frac{1}{\psi^{2}(x)}\right], \\
\mathscr{P}_{n, h}^{(d)}(t ; x)-x=\psi^{2}(x)\left[e^{-n x / b_{n}-h} \sum_{k=0}^{\infty} g_{k}^{d+1}\left(\frac{n x}{b_{n}}, h\right)\right. \\
\left.\cdot \frac{1}{\psi\left((k / n) b_{n}\right) k !} f\left(\frac{k}{n} b_{n}\right)-\frac{1}{\psi(x)}\right], \\
\mathscr{P}_{n, h}^{(d)}\left(\psi^{2} ; x\right)-\psi^{2}(x)=0 .
\end{gathered}
$$

From Lemma 2, we have

$$
\begin{aligned}
& \lim _{n \rightarrow \infty} \| e^{-n x / b_{n}-h} \sum_{k=0}^{\infty} g_{k}^{d+1}\left(\frac{n x}{b_{n}}, h\right) \\
& \cdot \frac{1}{\psi^{2}\left((k / n) b_{n}\right) k !} f\left(\frac{k}{n} b_{n}\right)-\frac{1}{\psi^{2}(x)} \|_{\eta}=0 \\
& \lim _{n \rightarrow \infty} \| e^{-n x / b_{n}-h} \sum_{k=0}^{\infty} g_{k}^{d+1}\left(\frac{n x}{b_{n}}, h\right) \\
& \cdot \frac{1}{\psi\left((k / n) b_{n}\right) k !} f\left(\frac{k}{n} b_{n}\right)-\frac{1}{\psi(x)} \|_{\psi}=0 .
\end{aligned}
$$

Using Lemma 2 and (31), we obtain

$$
\begin{gathered}
\left\|\mathscr{P}_{n, h}^{(d)}(1 ; x)-1\right\|_{\psi^{2} \eta}=\lim _{n \rightarrow \infty} \| e^{-n x / b_{n}-h} \sum_{k=0}^{\infty} g_{k}^{d+1}\left(\frac{n x}{b_{n}}, h\right) \\
\cdot \frac{1}{\psi^{2}\left((k / n) b_{n}\right) k !} f\left(\frac{k}{n} b_{n}\right)-\frac{1}{\psi^{2}(x)} \|_{\eta} \leq(2 h(d \\
+1)+1) \frac{b_{n}}{n}+\left(\frac{b_{n}}{n}\right)^{2} h(h+1)(d+1)^{2}=\alpha_{n} .
\end{gathered}
$$

By means of Lemma 2 and (32), one gets

$$
\begin{aligned}
& \left\|\mathscr{P}_{n, h}^{(d)}(\psi ; x)-\psi\right\|_{\psi^{2} \eta} \\
& =\lim _{n \rightarrow \infty} \| e^{-n x / b_{n}-h} \sum_{k=0}^{\infty} g_{k}^{d+1}\left(\frac{n x}{b_{n}}, h\right) \\
& \cdot \frac{1}{\rho\left((k / n) b_{n}\right) k !} f\left(\frac{k}{n} b_{n}\right)-\frac{1}{\psi(x)} \|_{\eta} \leq \frac{b_{n}}{n} h(d+1) \\
& =\beta_{n} .
\end{aligned}
$$

Finally, from (33), we obtain

$$
\left\|\mathscr{P}_{n, h}^{(d)}\left(\psi^{2} ; x\right)-\psi^{2}\right\|_{\psi^{2} \eta}=0=\gamma_{n} .
$$

If we apply Theorem 10, we obtain the desired result.

\section{A-Statistical Convergence}

Now, let $A=\left[a_{j n}\right], j, n \in \mathbb{N}$, be an infinite summability matrix. For a given sequence $\left(x_{n}\right)$, the $A$-transform of $x$, denoted by $\left((A x)_{j}\right)$, is given by

$$
(A x)_{j}=\sum_{n=1}^{\infty} a_{j n} x_{n}
$$

which provides the converging series for each $j \in \mathbb{N}$. We say that $A$ is a regular if $\lim _{j}(A x)_{j}=L$ whenever $\lim _{n}\left(x_{n}\right)=L$. A sequence $\left(x_{n}\right)$ is called $A$-statistically convergent to $L$ if, for every $\epsilon>0, \lim _{j} \sum_{n:\left|x_{n}-L\right| \geq \epsilon} a_{j n}=0$. This limit is denoted by 
$s t_{A}-\lim _{n} x_{n}=L$. Replacing $A$ by $C_{1}$, the Cesàro matrix of order one in (6), from $A$-statistical convergence, is reduced to statistical convergence. Similarly, if we take $A=I$, the identity matrix, then $A$-statistical convergence coincides with the ordinary convergence. Kolk [13] proved that, in the case of $\lim _{j} \max _{n}=0, A$-statistical convergence is stronger than ordinary convergence.

Further, we will first obtain the following weighted Korovkin theorem via $A$-statistical convergence.

Theorem 12. Let $\left(a_{n k}\right)$ be a nonnegative regular infinite summability matrix and $x \in[0, \infty)$. Let $\psi_{\gamma} \geq 1$ be a continuous function such that

$$
\lim _{n \rightarrow \infty} \psi(x) \psi_{\gamma}^{-1}(x)=0
$$

Then, for all $f \in C_{\psi}^{*}[0, \infty)$, we have

$$
s t_{A}-\lim _{n \rightarrow \infty}\left\|\mathscr{G}_{n, h}^{(d)}(f ; x)-f\right\|_{\psi_{\gamma}}=0 .
$$

Proof. From [14], for any $f \in C_{\psi}^{*}[0, \infty)$, it is enough to prove that

$$
s t_{A}-\lim _{n \rightarrow \infty}\left\|\mathscr{G}_{n, h}^{(d)}\left(e_{i} ; x\right)-e_{i}\right\|_{\psi_{\gamma}}=0,
$$

$$
\text { for } e_{i}=t^{i}, i=0,1,2 .
$$

So, by Lemma 2, we can easily get

$$
s t_{A}-\lim _{n \rightarrow \infty}\left\|\mathscr{G}_{n, h}^{(d)}\left(e_{0} ; x\right)-e_{0}\right\|_{\psi_{\gamma}}=0
$$

Again, by using Lemma 2, we have

$$
\begin{aligned}
\left\|\mathscr{G}_{n, h}^{(d)}\left(e_{1} ; x\right)-e_{1}\right\|_{\psi} & =\frac{b_{n}}{n} h(d+1) \sup _{x \in[0, \infty)} \frac{1}{1+x^{2}} \\
& \leq \frac{b_{n}}{n} h(d+1) .
\end{aligned}
$$

Now, for every given $\epsilon>0$, let us define the following sets:

$$
\begin{aligned}
S & =\left\{n:\left\|\mathscr{G}_{n, h}^{(d)}\left(e_{1} ; x\right)-e_{1}\right\|_{\psi} \geq \epsilon\right\}, \\
S_{1} & =\left\{n: \frac{b_{n}}{n} h(d+1) \geq \epsilon\right\} .
\end{aligned}
$$

It is clear that $S \subseteq S_{1}$. Hence, for all $n \in N$, we get

$$
\sum_{k \in S} a_{n k} \leq \sum_{k \in S_{1}} a_{n k}
$$

Therefore, $s t_{A}-\lim _{n \rightarrow \infty}\left\|\mathscr{G}_{n, h}^{(d)}\left(e_{1} ; x\right)-e_{1}\right\|_{\psi_{\gamma}}=0$.
Similarly, we have

$$
\begin{aligned}
& \left\|\mathscr{G}_{n, h}^{(d)}\left(e_{2} ; x\right)-e_{2}\right\|_{\psi} \\
& =\sup _{x \in[0, \infty)} \frac{x}{1+x^{2}} \frac{b_{n}}{n}(2 h(d+1)+1) \\
& \quad+\frac{b_{n}^{2}}{n^{2}} h(h+1)(d+1)^{2} \sup _{x \in[0, \infty)} \frac{1}{1+x^{2}} \\
& \leq(2 h(d+1)+1) \frac{b_{n}}{n}+\left(\frac{b_{n}}{n}\right)^{2} h(h+1)(d+1)^{2} .
\end{aligned}
$$

Now, we define the following sets:

$$
\begin{aligned}
U & =\left\{n:\left\|\mathscr{G}_{n, h}^{(d)}\left(e_{2} ; x\right)-e_{2}\right\|_{\psi} \geq \epsilon\right\}, \\
U_{1} & =\left\{n:(2 h(d+1)+1) \frac{b_{n}}{n} \geq \frac{\epsilon}{2}\right\}, \\
U_{2} & =\left\{n:\left(\frac{b_{n}}{n}\right)^{2} h(h+1)(d+1)^{2} \geq \frac{\epsilon}{2}\right\} .
\end{aligned}
$$

In view of (46), it is clear that $U \subseteq U_{1} \cup U_{2}$, which yields

$$
\sum_{k \in U} a_{n k} \leq \sum_{k \in U_{1}} a_{n k}+\sum_{k \in U_{2}} a_{n k} .
$$

Thus, we get $s t_{A}-\lim _{n \rightarrow \infty}\left\|\mathscr{G}_{n, h}^{(d)}\left(e_{2} ; x\right)-e_{2}\right\|_{\psi_{\gamma}}=0$.

Similarly, from Lemma 3, we have

$$
s t_{A}-\lim _{n \rightarrow \infty}\left\|\eta_{n, s}^{(d)}(x)\right\|_{\psi}=0, \quad s=1,2,3,4 .
$$

Now, we give a Voronovskaja type relation for the operators $\mathscr{G}_{n, h}^{(d)}$.

Theorem 13. Let $A=\left(a_{n k}\right)$ be a nonnegative regular infinite summability matrix. Then, for every $f \in C_{\psi}^{*}[0, \infty)$ such that $f^{\prime}, f^{\prime \prime} \in C_{\psi}^{*}[0, \infty)$, one has

$$
\begin{aligned}
s t_{A} & -\lim _{n \rightarrow \infty} \frac{n}{b_{n}}\left(\mathscr{G}_{n, h}^{(d)}(f ; x)-f(x)\right) \\
& =h(d+1) f^{\prime}(x)+\frac{x}{2} f^{\prime \prime}(x)
\end{aligned}
$$

uniformly with respect to $x \in[0, E],(E>0)$.

Proof. Let $x \geq 0$, and $f^{\prime}, f^{\prime \prime} \in C_{\psi}^{*}[0, \infty)$. Define the function $\theta$ by

$$
\theta(t, x)= \begin{cases}\frac{f(t)-f(x)-(t-x) f^{\prime}(x)-(1 / 2)(t-x)^{2} f^{\prime \prime}(x)}{(t-x)^{2}}, & \text { if } t \neq x, \\ 0, & \text { if } t=x\end{cases}
$$


Then, by assumption, we get $\theta(x, x)=0$ and $\theta(\cdot, x) \in C_{\psi}^{*}[0$, $\infty)$. obtain

By the linearity of $\mathscr{G}_{n, h}^{(d)}$ applied to the last equality, we

$$
\begin{aligned}
\frac{n}{b_{n}}\left(\mathscr{G}_{n, h}^{(d)}(f ; x)-f(x)\right) & \\
= & \frac{n}{b_{n}} \eta_{n, 1}^{(d)}(x) f^{\prime}(x)+\frac{1}{2} \frac{n}{b_{n}} \eta_{n, 2}^{(d)}(x) f^{\prime \prime}(x) \\
& +\frac{n}{b_{n}} \mathscr{G}_{n, h}^{(d)}\left(\theta(t, x)(t-x)^{2} ; x\right) .
\end{aligned}
$$

In view of Lemma 2, we get

$$
\begin{aligned}
& s t_{A}-\lim _{n \rightarrow \infty} \frac{n}{b_{n}} \eta_{n, 1}^{(d)}(x)=h(d+1), \\
& s t_{A}-\lim _{n \rightarrow \infty} \frac{n}{b_{n}} \eta_{n, 2}^{(d)}(x)=x, \\
& s t_{A}-\lim _{n \rightarrow \infty} \frac{n^{2}}{b_{n}^{2}} \eta_{n, 4}^{(d)}(x)=3 x^{2} .
\end{aligned}
$$

For the last term of the right hand side in (52), using CauchySchwarz inequality, we are led to

$$
\begin{aligned}
& \frac{n}{b_{n}} \mathscr{G}_{n, h}^{(d)}\left(\theta(t, x)\left(e_{1}-x\right)^{2} ; x\right) \\
& \quad \leq \sqrt{\mathscr{G}_{n, h}^{(d)}\left(\xi^{2}(t, x) ; x\right)} \sqrt{\frac{n^{2}}{b_{n}^{2}} \eta_{n, 4}^{(d)}(x)} .
\end{aligned}
$$

We observe that $\theta^{2}(x, x)=0$, and $\theta^{2}(\cdot, x) \in C_{\gamma}^{*}[0, \infty)$. In view of Theorem 4,

$$
s t_{A}-\lim _{n \rightarrow \infty} \mathscr{G}_{n, h}^{(d)}\left(\theta^{2}(t, x) ; x\right)=\theta^{2}(x, x)=0 .
$$

Then, it follows from (32) that

$$
s t_{A}-\lim _{n \rightarrow \infty} \frac{n}{b_{n}} \mathscr{G}_{n, h}^{(d)}\left(\theta(t, x)\left(e_{1}-x\right)^{2} ; x\right)=0
$$

uniformly in $x \in[a, b]$. Combining (27), (31), and (46), we get the desired result.

Let $C_{B}[0, \infty)$ denote the space of all real-valued bounded and uniformly continuous functions $f$ on $[0, \infty)$, endowed with the norm $\|f\|=\sup _{x \in[0, \infty)}|f(x)|$. Further, the appropriate Peetre $K$-functional is given by

$$
K_{2}(f ; \delta)=\inf _{g \in W^{2}}\left\{\|f-g\|+\delta\left\|g^{\prime \prime}\right\|\right\},
$$

where $\Delta^{2}=\left\{g \in C_{B}[0, \infty): g^{\prime}, g^{\prime \prime} \in \widetilde{C}_{B}[0, \infty)\right\}$. By $[15$, Theorem 4] we can find a constant $C>0$ such that

$$
K_{2}(f ; \delta) \leq C \omega_{2}(f ; \sqrt{\delta})
$$

where $\omega_{2}(f ; \sqrt{\delta})$ is the second-order modulus of continuity of $f \in C_{B}[0, \infty)$. Now, we obtain the rate of $A$-statistical convergence for the operators $\mathscr{G}_{n, h}^{(d)}$ with the help of Peetre's $K$ functional.
Theorem 14. Let $f \in \Delta^{2}$. Then, one has

$$
s t_{A}-\lim _{n \rightarrow \infty}\left\|\mathscr{G}_{n, h}^{(d)}(f ; x)-f\right\|_{C_{B}[0, \infty)}=0 .
$$

Proof. By the linearity property of $\mathscr{G}_{n, h}^{(d)}$ operators and using the local Taylor formula to the function $f \in \Delta^{2}$, we have

$$
\begin{aligned}
\mathscr{G}_{n, h}^{(d)}(f ; x)-f(x)= & \eta_{n, 1}^{(d)}(x) f^{\prime}(x) \\
& +\frac{1}{2} \eta_{n, 2}^{(d)}(x) f^{\prime \prime}(\xi),
\end{aligned}
$$

where $\xi \in(t, x)$.

Thus, we get

$$
\begin{aligned}
& \left\|\mathscr{G}_{n, h}^{(d)}(f ; x)-f\right\|_{C_{B}[0, \infty)} \\
& =\left\|f^{\prime}\right\|_{C_{B}[0, \infty)}\left\|\eta_{n, 1}^{(d)}(x)\right\|_{C_{B}[0, \infty)} \\
& \quad+\frac{1}{2}\left\|f^{\prime \prime}\right\|_{C_{B}[0, \infty)}\left\|\eta_{n, 2}^{(d)}(x)\right\|_{C_{B}[0, \infty)} .
\end{aligned}
$$

In view of (49) for $\epsilon>0$, we have

$$
\begin{aligned}
& \lim _{n \in N: I_{1} \geq \epsilon / 2} a_{n k}=0, \\
& \lim _{n} \sum_{k \in N: I_{2} \geq \epsilon / 2} a_{n k}=0 .
\end{aligned}
$$

From (61), one can write the following:

$$
\begin{aligned}
& \quad \sum_{k \in N:\left\|\mathcal{G}_{n, h}^{(d)}(f ; x)-f\right\|_{C_{B}[0, \infty)}} a_{n k} \leq \sum_{k \in N: I_{1} \geq \epsilon / 2} a_{n k}+\sum_{k \in N: I_{2} \geq \epsilon / 2} a_{n k} \\
& =0 .
\end{aligned}
$$

Hence, taking the limit as $n \rightarrow \infty$, we get the desired result.

The following theorem contains quantitative estimates by means of Peetre's $K$-functional.

Theorem 15. Letting $f \in C_{B}[0, \infty)$, one has the estimate

$$
\left\|\mathscr{G}_{n, h}^{(d)}(f ; x)-f\right\|_{C_{B}[0, \infty)} \leq M \omega_{2}\left(f ; \sqrt{\delta_{n}}\right),
$$

where $\delta_{n}=\left\|\eta_{n, 1}^{(d)}(x)\right\|_{C_{B}[0, \infty)}+\left\|\eta_{n, 2}^{(d)}(x)\right\|_{C_{B}[0, \infty)}$.

Proof. Letting $g \in \Delta^{2}$, by (61), we have

$$
\begin{aligned}
& \left\|\mathscr{G}_{n, h}^{(d)}(f ; x)-f\right\|_{C_{B}[0, \infty)} \\
& =\left\|f^{\prime}\right\|_{C_{B}[0, \infty)}\left\|\eta_{n, 1}^{(d)}(x)\right\|_{C_{B}[0, \infty)} \\
& \quad+\frac{1}{2}\left\|f^{\prime \prime}\right\|_{C_{B}[0, \infty)}\left\|\eta_{n, s}^{2}(x)\right\|_{C_{B}[0, \infty)} \\
& \leq\left\{\left\|\eta_{n, 1}^{(d)}(x)\right\|_{C_{B}[0, \infty)}+\frac{1}{2}\left\|\eta_{n, 2}^{(d)}(x)\right\|_{C_{B}[0, \infty)}\right\}\|f\|_{\Delta^{2}} .
\end{aligned}
$$


Using the last inequality for $f \in C_{B}[0, \infty)$, and $g \in \Delta^{2}$, we get

$$
\begin{aligned}
\left\|\mathscr{G}_{n, h}^{(d)}(f ; x)-f\right\|_{C_{B}[0, \infty)} & \\
= & \left\|\mathscr{G}_{n, h}^{(d)}(f ; x)-\mathscr{G}_{n, h}^{(d)}(g)\right\|_{C_{B}[0, \infty)} \\
& \quad+\left\|\mathscr{G}_{n, h}^{(d)}(g ; x)-g\right\|_{C_{B}[0, \infty)}+\|g-f\|_{C_{B}[0, \infty)} \\
\leq & \|g-f\|_{C_{B}[0, \infty)}+\left\|\mathscr{G}_{n, h}^{(d)}(g ; x)-g\right\|_{C_{B}[0, \infty)} \\
\leq & 2\|g-f\|_{C_{B}[0, \infty)}+\delta_{n}\|g\|_{W^{2}} .
\end{aligned}
$$

Taking the infimum on the right sides of the above inequality for all functions $g \in \Delta^{2}$, so

$$
\left\|\mathscr{G}_{n, h}^{(d)}(f ; x)-f\right\|_{C_{B}[0, \infty)} \leq K_{2}\left(f ; \delta_{n}\right) .
$$

By using the relation between Peetre's $K$-functional and the second modulus of smoothness given in [15], we get

$$
\begin{aligned}
& \left\|\mathscr{G}_{n, h}^{(d)}(f ; x)-f\right\|_{C_{B}[0, \infty)} \\
& \quad \leq M\left\{\omega_{2}\left(f ; \sqrt{\delta_{n}}\right)+\min \left(1, \delta_{n}\right)\|g\|_{C_{B}[0, \infty)}\right\} .
\end{aligned}
$$

From (49), we get $s t_{A}-\lim _{n \rightarrow \infty} \delta_{n}=0$, and hence $s t_{A}-$ $\lim _{n \rightarrow \infty} \omega_{2}\left(f ; \sqrt{\delta_{n}}\right)=0$. Therefore, we get the rate of $A$ statistical convergence of the sequence $\mathscr{G}_{n, h}^{(d)}$ to $f(x)$ in the space $C_{B}[0, \infty)$.

\section{Conflicts of Interest}

The authors declare that they have no conflicts of interest.

\section{References}

[1] A. Jakimovski and D. Leviatan, "Generalized Szász operators for the approximation in the infinite interval," Mathematica-Revue d'Analyse Numérique et de Théorie de l'Approximation, vol. 11, no. 34, pp. 97-103, 1969.

[2] İ. Büyükyazıc1, H. Tanberkan, S. K. Serenbay, and Ç. Atakut, "Approximation by Chlodowsky type Jakimovski-Leviatan operators," Journal of Computational and Applied Mathematics, vol. 259, pp. 153-163, 2014.

[3] A. Aral and V. Gupta, "The $q$-derivative and applications to $q$ Szász Mirakyan operators," Calcolo. A Quarterly on Numerical Analysis and Theory of Computation, vol. 43, no. 3, pp. 151-170, 2006.

[4] A. Kajla and P. N. Agrawal, "Approximation properties of Szász type operators based on Charlier polynomials," Turkish Journal of Mathematics, vol. 39, no. 6, pp. 990-1003, 2015.

[5] A. Kajla and P. N. Agrawal, "Szász-Durrmeyer type operators based on Charlier polynomials," Applied Mathematics and Computation, vol. 268, pp. 1001-1014, 2015.

[6] A. Kajla and P. N. Agrawal, "Szász-Kantorovich type operators based on Charlier polynomials," Kyungpook Mathematical Journal, vol. 56, no. 3, pp. 877-897, 2016.
[7] F. Taşdelen, R. Aktaş, and A. Altin, "A Kantorovich type of Szasz operators including Brenke-type polynomials," Abstract and Applied Analysis, vol. 2012, Article ID 867203, 13 pages, 2012.

[8] N. I. Mahmudov, "Approximation by the $q$-Szász-Mirakjan operators," Abstract and Applied Analysis, vol. 2012, Article ID 754217, 16 pages, 2012.

[9] S. Sucu, G. Içöz, and S. Varma, "On some extensions of szasz operators including boas-buck-type polynomials," Abstract and Applied Analysis, vol. 2012, Article ID 680340, 15 pages, 2012.

[10] V. Gupta and R. P. Agarwal, Convergence Estimates in Approximation Theory, Springer, New York, NY, USA, 2014.

[11] F. Altomare and M. Campiti, Korovkin-Type Approximation Theory and Its Applications, Walter de Gruyter, New York, NY, USA, 1994.

[12] A. D. Gadjiev and A. Aral, "The estimates of approximation by using a new type of weighted modulus of continuity," Computers \& Mathematics with Applications. An International Journal, vol. 54, no. 1, pp. 127-135, 2007.

[13] E. Kolk, "Matrix summability of statistically convergent sequences," Analysis, vol. 13, no. 1-2, pp. 77-84, 1993.

[14] A. D. Gadjiev, “Theorems of the type of P. P. Korovkin's theorems," Akademiya Nauk SSSR. Matematicheskie Zametki, vol. 20, no. 5, pp. 781-786, 1976.

[15] R. A. DeVore and G. G. Lorentz, Constructive approximation, vol. 303 of Grundlehren der Mathematischen Wissenschaften [Fundamental Principles of Mathematical Sciences], SpringerVerlag, Berlin, Germany, 1993. 


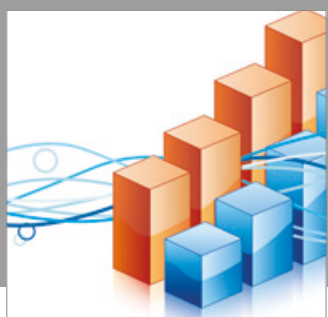

Advances in

Operations Research

vatersals

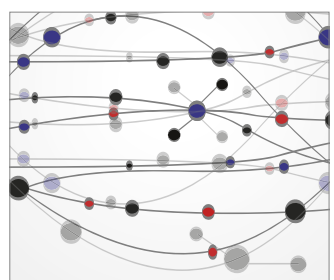

\section{The Scientific} World Journal
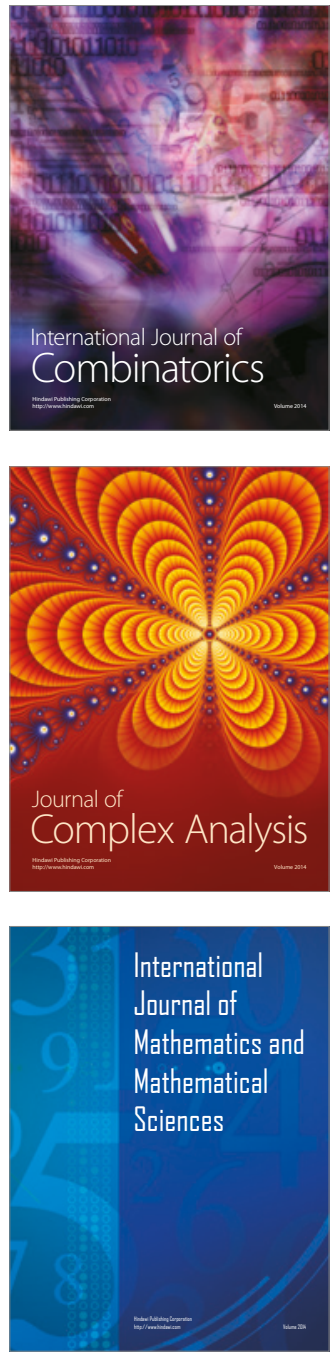
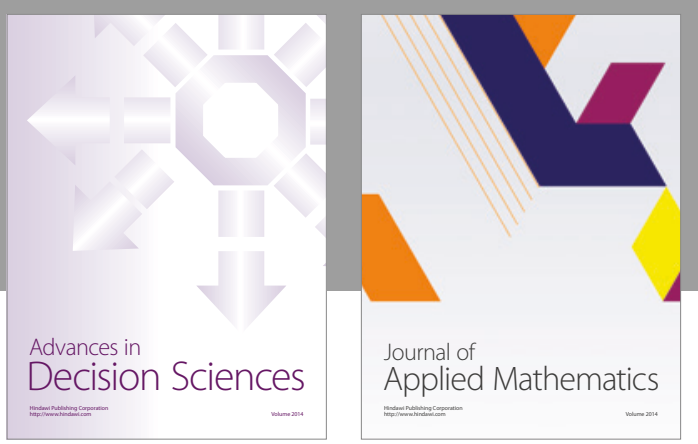

Algebra

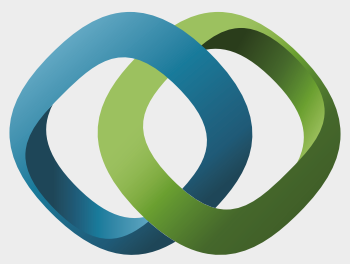

\section{Hindawi}

Submit your manuscripts at

https://www.hindawi.com
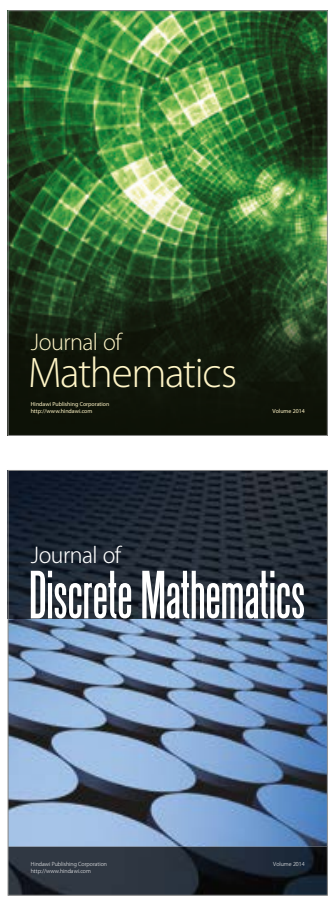

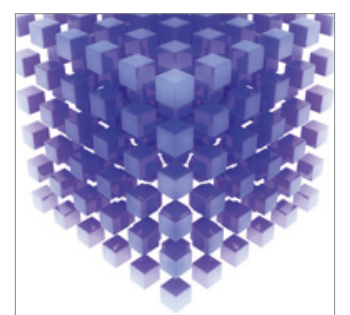

Mathematical Problems in Engineering
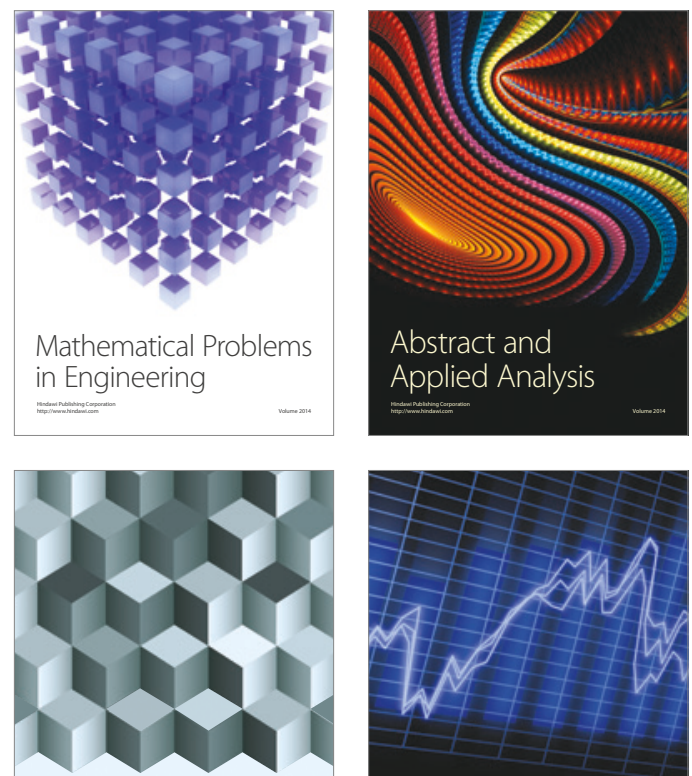

Journal of

Function Spaces

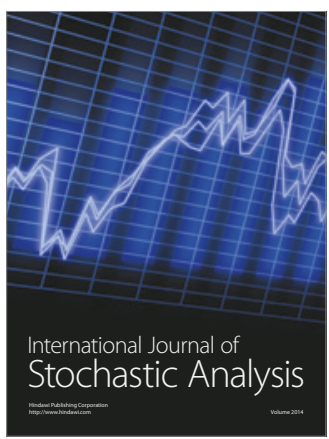

Probability and Statistics
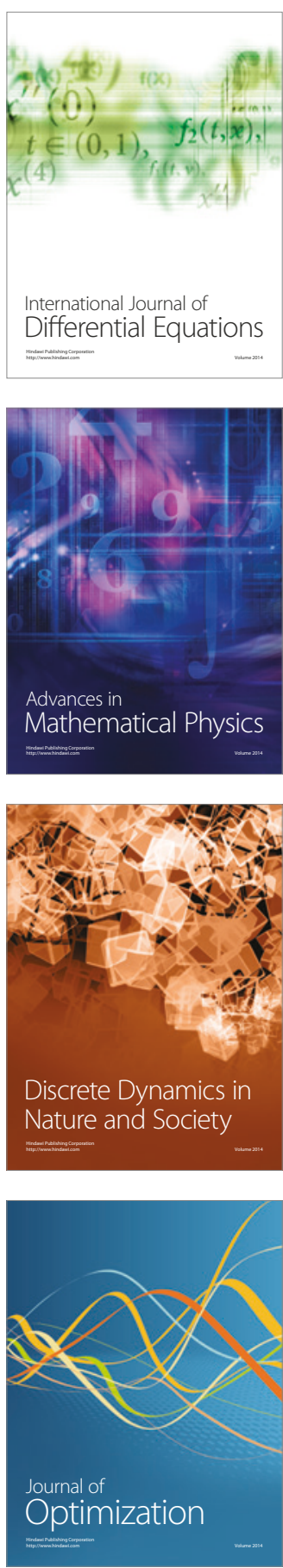